\title{
Effect and correction of the shift in spectral images for polychromatic thermography
}

\author{
R. Gilblas*,1, T. Sentenac \\ Université de Toulouse; CNRS, Mines Albi, INSA, UPS, ISAE-SUPAERO; ICA (Institut Clément Ader), Campus Jarlard, F-81013 Albi, France
}

\begin{abstract}
A B S T R A C T
This articles investigates the temperature errors due to chromatic aberration in multiwavelength thermography methods. The Chromatic aberration leads to a shift in the perspective projection of a point in the $3 D$ space on the image formed at different wavelengths. This shift causes an error in the temperature field calculated by polychromatic methods, from the fusion for each pixel of radiance temperature images at different wavelengths. The temperature error can reach $40 \%$ on a sample with high spatial nonuniformities, due to wide variations of emissivity. This paper suggests an approach to correcting the chromatic aberration that is based not on equipment but on software, coupled with a calibration, using Digital Image Correlation (DIC). This experimental technique is a 2D optical method used in mechanical engineering, for inferring a deformation of a plane structure's surface from a displacement field calculated by correlation between pixels of two successive images. This paper applies the technique to achieve the field displacement induced between two images at two wavelengths by chromatic aberration. After applying this correcting displacement field, the temperature error decreases from $40 \%$ to $1 \%$ for the pixels located at the boundary of two areas with different emissivities.
\end{abstract}

Keywords:

Thermography

Chromatic aberration

Multispectral imaging

Metrology

\section{Introduction}

Thermography is a widely-used technique today in numerous applications, such as defect detection ([3]), thermal properties characterization ([8]), temperature measurement $([13,21])$ or military surveillance [25]. In applications that require quantitative measurements of temperature fields, the influence of the emissivity, which is a thermo-optical property of the material, needs to be investigated. To bypass the need for an absolute knowledge of the emissivity, some techniques formulate assumptions and models of its spectral behaviour and solve, at different wavelengths, a system of equations using this modelling with images of radiance temperature. The number of equations depends on the parameters used in the emissivity modelling, and the choice of wavelengths can be critical [18]. Solving this system of equations from images of radiance temperature at different wavelengths is performed for each pixel to retrieve the true temperature and emissivity fields. For example, monochromatic thermography considers the emissivity as a known value. Bichromatic thermography [19] assumes

\footnotetext{
* Corresponding author.

E-mail address: rgilblas@mines-albi.fr (R. Gilblas).

1 www.institut-clement-ader.org.
}

that the emissivity ratio at two wavelengths is known. Polychromatic thermography [6] is based on different emissivity modelling versus wavelength (polynomial, exponential, etc.). Active bichromatic thermography [2] adds equations to take into account strong parasite reflections on a specular metallic surface. Bichromatic thermoreflectometry [7] evaluates the emissivity ratio at two wavelengths by a reflectometry measurement.

The implementation of these techniques relies on systems of spectral selection of the thermal radiation. Especially for bichromatic techniques, the use of dual-band cameras [1,17], is an attractive solution. These cameras usually operate both in the 8-12 $\mu \mathrm{m}$ Long Wavelength Infrared (LWIR) and the 3-5 $\mu \mathrm{m}$ Medium Wavelength Infrared (MWIR) spectral bands. Both operating spectral bands may be too far to be able to make any assumptions about the emissivity. Moreover, a spectral selection must often be added to reduce the bandwidth of each spectral band by using interference filters. Whether with dual or single band cameras, spectral selection is very often performed by the switching of interference filters. Unfortunately, interference filters on the lens introduce optical defects [10] like ghost and narcissus effects and chromatic aberration. These defects are widely presented in the literature [15].

Indeed, the optical index of the material of lenses and filters 
depends on the wavelength, and the optical paths for different wavelengths differ significantly. The perspective projection of a 3D point of the object on the focal plane of the detector array is then shifted from one wavelength to the other. The images are thus distorted in two components: lateral (shift in the images) and longitudinal (de-focussing). This paper only addresses the lateral distortion of the images at several wavelengths.

For polychromatic thermography techniques, based on the resolution of a system of equations with images at different wavelengths, their shift means that for a given pixel, the matching 3D point in the scene is not the same for different wavelengths. The resolution for each pixel is thus physically inconsistent, which induces an error in the temperature field calculated. A correction method needs to be implemented to ensure that the same 3D point is projected onto the same pixel regardless of the selected wavelength. The ideal method would be to include it in a prior calibration procedure that can be adapted to any system consisting of cameras, lenses and filters.

Many articles address the problem of the correction of chromatic aberration, but they deal mostly with the visible spectrum for colour cameras (where the RGB channels are shifted), and they often concern photogrammetry applications [14]. Chromatic aberration can initially be reduced by taking a few precautions, such as closing the aperture of the lens to a maximum so that only the centre of the lens is used. However, this is very restrictive for low flux applications. Alternatively, specific equipment can be added to limit the effect of chromatic aberration. Telecentric lenses [20] allow the rays to be turned perpendicularly to the detector plane array. The drawbacks to this method are that it gives a small depth of field and a significant length (around $50 \mathrm{~cm}$ ). Achromatic and apochromatic lenses [23], with a suitable surface treatment on the glass, enable the correction of optical paths for two or three wavelengths. In addition to their rather high prices, these components must be added to a lens with a compatible focal length, which restricts their use to fixed focal lenses and lowers the signal received by the detectors.

Another solution, easily adaptable to any system and which also removes the remaining defects of the previous solutions, is to correct the distortion directly on the images by image processing procedures. The first class of procedures benefits from the features of the scene under view. In Ref. [4], automatic detection of edges and contours is set up in the image, and image warping is applied. The second class of procedures includes the modelling of chromatic aberration in which parameters are estimated in a calibration step using a specific pattern [11,15]. These methods are dedicated to colour cameras which use a colour filter array consisting of a mosaic of spectral filters in front of the image sensor. The most common spectral filter array is the Bayer filter which alternates red and green filters for the odd pixel rows and green and blue filters for the even pixel rows of the image sensor. The image processing procedure [12] precisely determines the geometric distortions of the red and blue image pixels in comparison to the green reference pixel. For polychromatic thermography systems, spectral filters are not dedicated to individual pixels but to all the pixels. The chromatic aberration is not between pixels but between images acquired at different wavelengths.

The suggested procedure for image processing does not compute displacement between pixels for transferring the perspective projection of 3D points from the green pixel to the red or blue ones, but rather it computes a displacement field on the whole image so as to transfer the perspective projection of $3 \mathrm{D}$ points to the same pixel of two images acquired at two different wavelengths.

The displacement fields are calculated by the Digital Image Correlation (DIC) method, which is able to provide dense displacement fields. This method is usually used in mechanical engineering [24] to measure displacement fields between two images and thus derive strain fields. It is therefore assumed that the difference between the images comes only from the effect of the displacement field of the observed structure. In our case, this technique measures the displacement field of a surface of an image at a given wavelength with respect to a reference image at another wavelength. It is therefore assumed that any difference between the reference image and the distorted image comes only from the effect of the chromatic aberration between the two wavelengths.

The first part of this paper seeks to evaluate the temperature error due to chromatic aberration in a bichromatic thermography system. This study is performed on a specific sample, called the "two-emissivity sample", showing two areas with two very different emissivities. The paper then presents the suggested image processing procedure based on the DIC method, which is applied to calculate the displacement field between two images at two wavelengths acquired on a system composed of a lens and interference filters. The displacement field is determined within a simple calibration step and applied in-line to correct the distortion due to the chromatic aberration. Finally, the method is validated on the previous two-emissivity sample, showing a significant decrease in the temperature error at the boundary of the two areas.

The paper is organized as follows. Section 2 is dedicated to the evaluation of the effects of the bichromatic thermography system. Section 3 provides the measurement of the image shift at different wavelengths by DIC methods implemented in the commercial software, Vic2D. Section 4 presents the correction protocol and shows the gains in terms of accuracy in temperature measurement.

\section{Temperature error of bichromatic thermography due to chromatic aberration}

\subsection{Principle of bichromatic thermography}

Bichromatic thermography is a non-contact temperature measurement method which does not require the absolute knowledge of emissivity; only the ratio at two wavelengths is necessary. The standard system is composed of a camera equipped with two interference filters at wavelengths $\lambda_{1}$ and $\lambda_{2}$. Two radiance temperature fields $T_{R 1}(u, v)$ and $T_{R 2}(u, v)$ can easily be calculated for each pixel of coordinates $(u, v)$ from two images acquired at the two wavelengths using a previous radiometric calibration performed on a blackbody for both filters (see article [22]). Knowing the radiance temperature fields and the emissivity ratio field $\varepsilon_{r}(u, v)$ of the scene under view, the colour temperature field $T_{C}(u, v)$ is calculated according to Eq. (1).

$$
\begin{aligned}
& \frac{1}{T_{C}(u, v)}=\frac{1}{T_{r}(u, v)}+\frac{\Lambda}{C_{2}} \ln \varepsilon_{r}(u, v) \\
& \frac{1}{T_{r}(u, v)}=\Lambda\left(\frac{1}{\lambda_{1} T_{R 1}(u, v)}-\frac{1}{\lambda_{2} T_{R 2}(u, v)}\right) \\
& \varepsilon_{r}(u, v)=\frac{\varepsilon_{1}(u, v)}{\varepsilon_{2}(u, v)}, \quad \Lambda=\frac{\lambda_{1} \lambda_{2}}{\lambda_{2}-\lambda_{1}}
\end{aligned}
$$

The formulation of the Eq. (1) is based on Wien's approximation (available when $\lambda T \leq 3000 \mu \mathrm{m} K$ and suitable for $\lambda \in$ [0.9-1.7 $\mu \mathrm{m}]$ spectral and $T \in\left[300-1000{ }^{\circ} \mathrm{C}\right]$ thermal range) and with assumptions of no reflected radiation on the sample and no atmospheric attenuation.

Obviously, this equation shows that the colour temperature field comes from the fusion, at each pixel, of four physical quantities: the radiance temperature fields and the emissivity fields at two wavelengths. These four quantities must be spatially coherent. A 
shift, even of a single pixel, between these quantities leads to a false solution of the equation for each pixel and an temperature field. This shift is even more damaging if the scene exhibits strong spatial non-uniformities of heat gradient and/or emissivity. Potential differential spatial distortions induced in each quantity by chromatic aberrations must be checked, quantified and corrected if needed.

\subsection{Calculation of the error on the "two-emissivity sample"}

To highlight an error in the calculated temperature field, a relevant non-uniform sample was designed, called a "two-emissivity sample", with a sharp boundary and very different emissivities for each homogeneous part. This sample, shown in Fig. 1c), is composed of Zirconia stabilized with yttria, which is doped with Erbium oxide ions on its left side, and with Dysprosium oxide ions on its right. These two parts, called YSZ:Er ${ }^{3+}$ and YSZ:Dy ${ }^{3+}$ respectively, have very different emissivity values and spectral variations, as determined in the paper [7]. This sample is heated uniformly on the rear side, so the surface temperature on the front side should be relatively homogeneous.

The bichromatic thermography is performed at two near wavelengths which are $\lambda_{1}=1.31 \mu \mathrm{m}$ and $\lambda_{2}=1.55 \mu \mathrm{m}$. The choice of these values is dictated by the requirement that they must 1) not be too close together to avoid parameter $\Lambda$ being too high, 2) not be too far apart, to stay in the detection spectral band (NIR $[0.9-1.7] \mu \mathrm{m})$ of the camera. It will be shown that, even for those relatively close wavelengths, the effect of chromatic aberration can

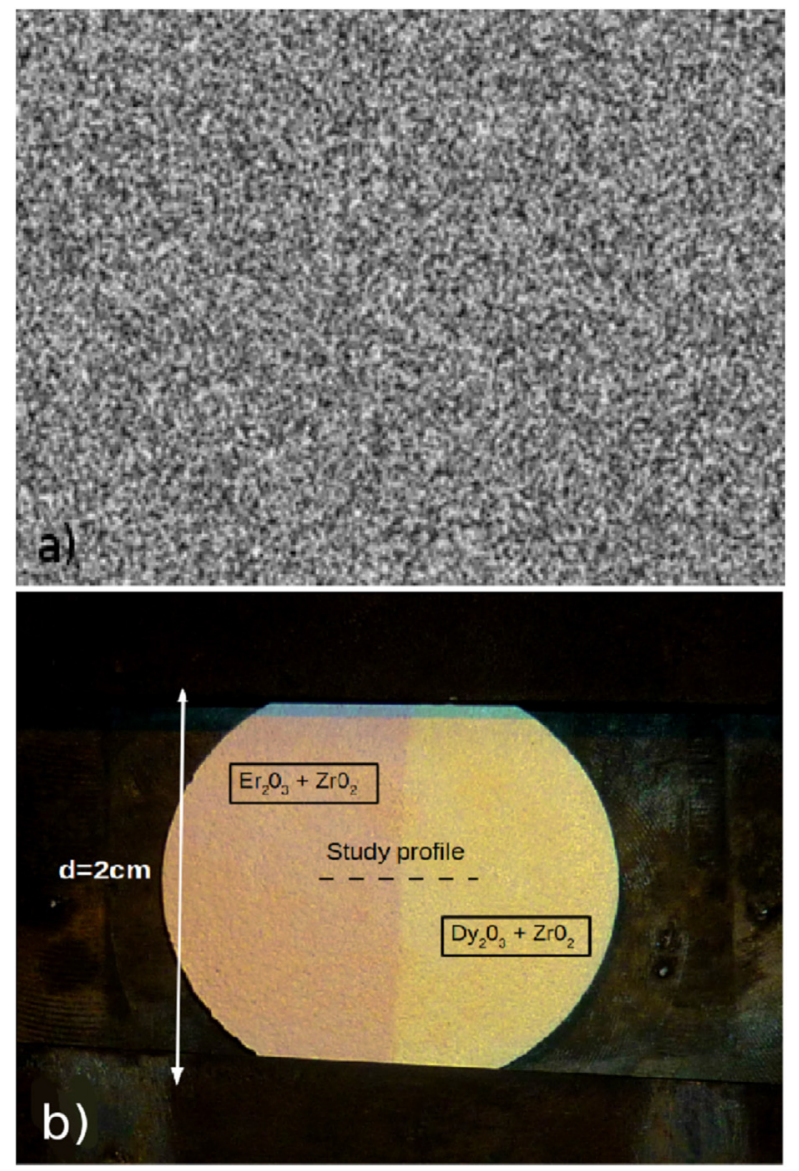

Fig. 1. Photograph of the scenes under study: a) Reference pattern (Perlin noise) for the distortion calculation by DIC; b) "Two-emissivity" sample for the temperature error evaluation (and the profile under study). be important.

This study of the temperature error is restricted to the profile following the centre dashed line shown in Fig. 1b). The radiance temperature profiles at two wavelengths $\lambda_{1}$ and $\lambda_{2}$ are drawn in Fig. 2. The radiance temperature $T_{R 1}$ is equal to $890 \mathrm{~K}$ in the $Y S Z: E r^{3+}$ part (from pixel 150 to 163 ) (and respectively $925 \mathrm{~K}$ in the $Y S Z: D y^{3+}$ part (from pixel 164 to 180 )). $T_{R 2}$ is equal to $930 \mathrm{~K}$ in the YSZ: $\mathrm{Er}^{3+}$ part (from pixel 150 to 166 ) (and respectively $905 \mathrm{~K}$ in the $Y S Z: D y^{3+}$ part (from pixel 167 to 180$)$ ). Their values are very different and cross over at the boundary, which testifies that the emissivity is very different from one part of the sample to the other, and that it increases versus wavelength for $Y S Z: \mathrm{Er}^{3+}$ and decreases for YSZ:Dy ${ }^{3+}$.

The radiance temperature value changes between pixels 163 and 164 , whereas the value of the radiance temperature $T_{R 2}$ changes between pixels 166 and 167. A shift is then recorded from one wavelength to the other of three pixels. Knowing the value and the shift of radiance temperatures and the value of the emissivity ratio $\varepsilon_{r}(u, v)$ measured by thermoreflectometry in a previous work (see article [7]), $\varepsilon_{r}=0.56$ of part YSZ:Er ${ }^{3+}$ (and respectively $\varepsilon_{r}=1.2$ of YSZ:Dy $\left.{ }^{3+}\right)$, the colour temperature $T_{C}(u, v)$ is calculated according to Eq. (1) and drawn in Fig. 2. For this correction, the boundary is selected between pixels 164 and 165 . On its left part, $\varepsilon_{r}=0.56$ is applied, and on the right part, $\varepsilon_{r}=1.2$.

Outside of the boundary between the two parts of the sample, from pixel 150 to 163 (for YSZ:Er ${ }^{3+}$ part), the value of the colour temperature is equal to $975 \mathrm{~K}$ (and respectively from pixel 167 to 180 (for YSZ: $D y^{3+}$ part), it is around $950 \mathrm{~K}$ ). The calculation of the colour temperature $T_{C}$ is therefore efficient (since the temperature is higher). The remaining difference between the two parts comes from the thermal conductivity differences (see [7]) between each part, i.e. the thermodynamic surface temperature is not exactly the same.

However, within the boundary between the two parts of the sample, from pixel 164 to 166 where the values of radiance temperature are shifted, the calculation of $T_{C}$ exhibits absurd values which lead to an error of $350 \mathrm{~K}$ for the worst case. The shift of radiance temperature at two wavelengths due to chromatic aberration leads to incoherent physical values (almost $40 \%$ of relative error on colour temperature) and a false interpretation of the colour temperature values. This shift, caused by lateral chromatic aberrations, is critical and it must be taken into account in

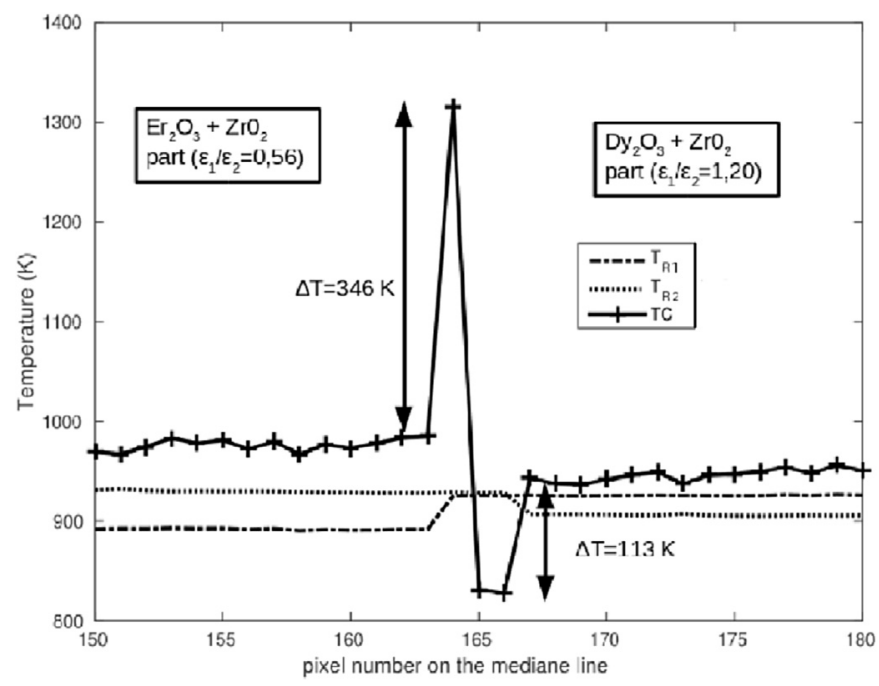

Fig. 2. Radiance and colour temperature on a profile of the "two-emissivity sample". 
polychromatic thermography techniques, particularly on heterogeneous scenes, for accurate quantitative measurement of temperatures fields.

\section{DIC measurement of the displacement map caused by lateral chromatic aberration}

Experimental evaluation of the lateral displacement induced by chromatic aberration is provided in this section.

\subsection{Background on Digital Image Correlation (DIC) for strain determination}

Digital Image Correlation (DIC) is usually used in mechanical engineering $([5,16])$ to measure displacement fields between two images and thus derive strain fields. It is therefore assumed that the difference between the images comes only from the effect of the displacement field of the observed structure. A $3 D$ point of physical coordinates $(x, y, z)$ then becomes the point $\left(x^{*}, y^{*}, z^{*}\right)$ after displacement. Through the $2 D$ perspective projection $(\mathscr{P})$ and following the Eq. (2), these $3 D$ points are projected into the pixel of coordinates $(u, v)$ and into the pixel of coordinates $(u+d u, v+d v)$ after displacement. $d u$ and $d v$ are then the apparent displacement in the image. According to assumptions of plane-displacement on the object and a previous geometric calibration, the displacement on the object can be retrieved.

$$
\begin{aligned}
(u, v) & =\mathscr{P}(x, y, z) \\
(u+d u, v+d v) & =\mathscr{P}\left(x^{*}, y^{*}, z^{*}\right)
\end{aligned}
$$

For the calculation of the apparent image displacement from a reference to a distorted image, $d u, d v$, the DIC method assumes that the grey level of the pixels between the images, acquired during the displacement, is preserved and might change slightly. The detection, tracking and matching of the grey level of a pixel in a sequence of images are performed according to different formulations of the equation of conservation of grey levels between a reference image and a distorted image (see [9]). The minimization of this conservation function is facilitated with images from an object which has been painted with a Perlin noise pattern, called "mouchetis". This pattern consists of randomly distributed grey levels with a good quality applied on the object. The size of the pattern must be compatible with the value of the displacement. The quality of this matching is represented by the correlation criterion, which indicates the areas on the image where DIC is successful or fails. The precision announced by the developers of VIC2D software on deformation is $\pm 0.02 \%$.

\subsection{Digital Image Correlation (DIC) method for lateral chromatic aberration determination}

This section provides the principle, the application and the tunable parameters of the method of DIC applied to a bichromatic thermography system.

\subsubsection{Principle of DIC measurement of displacement induced by chromatic aberration}

The displacement field is then measured between a reference image at a given wavelength and a distorted image at another wavelength. It is assumed that any difference between the reference and the distorted image comes from the sole effect of the chromatic aberration between the two wavelengths. This approach introduces two main differences with respect to the mechanical engineering application. First, the physical phenomenon at the origin of the displacement is completely different. In mechanical experiments, the displacement field is provided by a strain on the material of the object. For chromatic aberration experiments, the displacement field comes from optical distortion introduced by the optical components of the camera (lens and interference) and not from physical phenomena acting on the object. The $3 D$ point on the object does not move, but it is the perspective projection matrix that induces distortion of the image, as depicted by Eq. (3) below:

$$
\begin{aligned}
(u, v) & =\mathscr{P}_{\lambda_{1}}(x, y, z) \\
\left(u+d u_{\lambda_{1} \rightarrow \lambda_{2}}, v+d v_{\lambda_{1} \rightarrow \lambda_{2}}\right) & =\mathscr{P}_{\lambda_{2}}(x, y, z)
\end{aligned}
$$

In the equation, the perspective projection matrix $\mathscr{P}_{\lambda}$ depends on the wavelength. The scene under view is a random pattern, of Perlin-noise-type, with a greyscale that varies continuously between the two extreme values set and with known sizes of the pattern. Reference calibration targets are thus manufactured with a calibrated Perlin noise pattern, contrast and size, depending on the displacement to be measured, as illustrated in Fig. 1a).

\subsubsection{Application of DIC to bichromatic thermography}

Digital Image Correlation is applied to a bichromatic thermography system composed of a camera equipped with a lens and interference filters for spectral selection. Two optical components induce chromatic aberrations and distortions between the bichromatic images. The displacement field is then calculated by the Digital Image Correlation method (using VIC2D commercial software) between the bichromatic images acquired in front of the reference calibration target and according to the wavelengths and the spectral bandwidths of the filter. The size of the global displacement is evaluated by the criterion $C$ which calculates the mean value of the displacement image, as written in Eq. (4):

$$
C=\sqrt{\frac{1}{M N} \sum_{u \prime=1}^{M} \sum_{\nu \prime=1}^{N}\left(d u_{\lambda_{1} \rightarrow \lambda_{2}}^{2}(u \prime, v \prime)+d v_{\lambda_{1} \rightarrow \lambda_{2}}^{2}(u \prime, v \prime)\right)}
$$

$N$ (and respectively $M$ ) is the number of rows (and respectively the number of columns) of the displacement image. Note that this size can be different from the image size, depending on the correlation window size and step. A high value of $C$ means a high value of the displacement over the whole image, i.e. a strong influence of lateral chromatic aberration.

\subsubsection{DIC tunable parameters}

DIC depends on the correlation window size (i.e. the search area to match the pattern between two images), and the step (i.e. the number of pixels between two consecutive correlation windows). The correlation window and the step thus define the displacement number calculated over the whole image. For example, choosing a correlation window of 25 pixels and a step of 10 pixels, reduces the number of points by a factor of 25 . A low correlation window size decreases the quality (or correlation criterion) of DIC. A compromise must be made to keep the number of points significant, and the quality of DIC acceptable. For our application, the default setting of a window size of 21 pixels and a step of 5 pixels seems compatible with the pattern's image and the quality of DIC.

\subsection{Experimental set-up}

The experimental set-up, based on a camera equipped with a lens and a filter wheel, on different active sources and on the reference calibration target, is presented as a schematic view in Fig. 3.

The Xenics Xeva NIR camera uses an InGaAs detector, enabling detection from 0.9 to $1.7 \mu \mathrm{m}$. The size of the matrix is $320 \times 256$ 


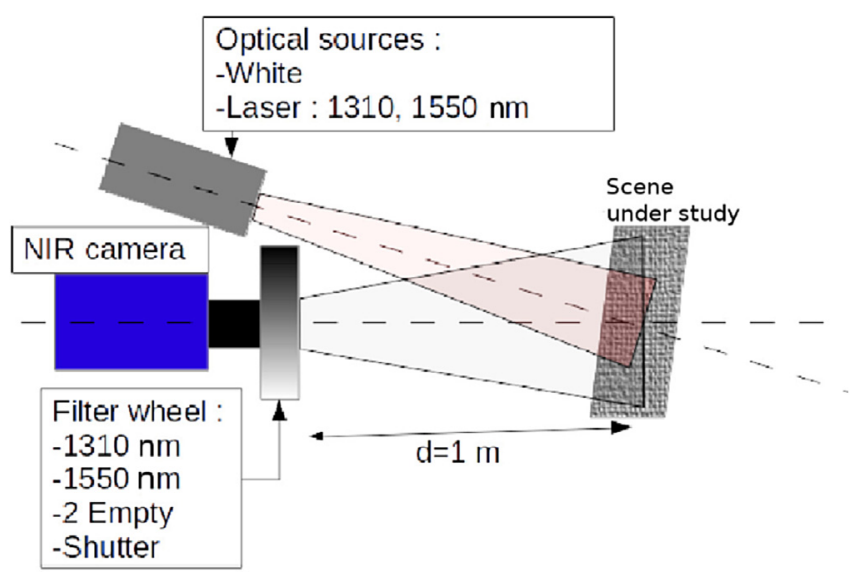

Fig. 3. Schematic top view of the apparatus.

with a pitch of $30 \mu \mathrm{m}$ and the integration time goes from $1 \mu \mathrm{s}$ to $1 \mathrm{~s}$.

The Sutter Instrument Filter wheel has 5 locations. 2 are occupied by 1310 and $1550 \mathrm{~nm}$ filters with a spectral bandwidth of $50 \mathrm{~nm}$. The optical sources are, firstly a standard $100 \mathrm{~W}$ Tungsten lamp generating a wide white spectrum over the visible and the NIR range, then two extended laser sources at wavelengths of 1310 and $1550 \mathrm{~nm}$ with a bandwidth of about $1 \mathrm{~nm}$. The beam diameters are about $5 \mathrm{~cm}$.

The reference target is a Perlin noise reference plate (Fig. 1a)) for the calculations of spectral displacements.

\subsection{Measurement of the displacement fields by DIC}

The different setup configurations are presented, and the determination of the displacements (field and mean values) are provided.

\subsubsection{Selection of configurations}

Five configurations were selected and are given in Table 1. Configuration 1 investigates the influence of the filter and the lens over a wide bandwidth $50 \mathrm{~nm}$. The reference calibration target is illuminated by the white source. The bichromatic images are the reference image acquired at the wavelength of $1.31 \mu \mathrm{m}$ and the distorted image acquired at the wavelength of $1.55 \mu \mathrm{m}$. This configuration is compared to configuration 2 to analyse the influence of the filter bandwidth on the chromatic aberration. In configuration 2 , the reference calibration target is then illuminated by the laser sources with a spectral bandwidth of $1 \mathrm{~nm}$. Configuration 3 highlights the influence of the lens on the chromatic aberration. The reference calibration target is still illuminated by the laser sources and the reference and distorted images are acquired without placing any filter in the optical path. Configurations 4 and 5 aim to evaluate the influence of the interference filter at each wavelength. In configuration 4 , the reference calibration target is illuminated by the laser source at $1.31 \mathrm{~m}$. The reference image at the wavelength of $1.31 \mu \mathrm{m}$ is acquired without a filter, and the distorted image with a filter. Configuration 5 is the same, but with a wavelength of $1.55 \mu \mathrm{m}$.

\subsubsection{Analysis of the mean value of the displacement}

In the last column of Table 1, the value of the criterion $C$ for the different configurations is displayed. The lowest value of $C(0.33)$ is provided by configuration 3 , which uses a short bandwidth source and does not include a filter in the optical path. The chromatic aberration that is due only to the lens is low, because the specific NIR lens is well adapted to the spectral response of the detector. The comparison of the value of $C$ between configurations 1 and 2 leads to the conclusion that the spectral bandwidth has no influence on chromatic aberration. These configurations correspond to the highest values of $C$, when the optical path includes both the lens and filters. Therefore, the major contribution to chromatic aberration appears to come from the filters.

The value of $C$ for configuration 1 is around the double than for configuration 4 or 5 . It seems that $C$ is roughly proportional to the number of filters involved in the calibration process. To compare the chromatic aberration introduced by each filter, configurations 4 and 5 are compared. The value of criterion $C$ is equal to 1.47 for configuration 4 and wavelength $1.31 \mu \mathrm{m}$ (and respectively, for configuration 5, 1.80 and wavelength $1.55 \mu \mathrm{m}$ ). The influence of the chromatic aberration is thus higher for the filter with a central wavelength of $1.55 \mu \mathrm{m}$.

\subsubsection{Analysis of the displacement fields}

Fig. 4 shows displacement fields for various configurations.

The lowest value of $C(0.33)$ recorded for configuration 3 corresponds to the displacement field displayed in Fig. 4b). This displacement field is a centred pattern. The vectors located in the centre of the field are almost null, and the vectors located on the borders of the field are directed towards the centre. This pattern is very comparable to the typical chromatic aberration encountered for visible lenses (distortion). For the central pixel, chromatic aberration induces an axial displacement of the focal point (simple blur). For the other pixels, chromatic aberration induces both blur and lateral shift. This lateral shift is all the more pronounced as the angle to this pixel of the central ray and the optical axis is high.

The highest value of $C$ (2.92) corresponds to Fig. 4a) which shows the displacement field resulting from the sum of the distortion induced by the lens and filter. The displacement field is calculated referring to wavelength $1.31 \mu \mathrm{m}$. For configuration 2, the displacement field is very similar to Fig. 4a). This confirms that a bandwidth of $50 \mathrm{~nm}$ or $1 \mathrm{~nm}$ has no influence on the distortion of the image.

Fig. 4c) and d) show displacement vectors aligned, pointing towards the top of the image for wavelength $1.31 \mu \mathrm{m}$ and towards the bottom right for wavelength $1.55 \mu \mathrm{m}$. This effect is a composition of the influence of the lens and the filter for each wavelength.

To conclude, the quantification of chromatic aberration was

Table 1

Description of the configurations and results.

\begin{tabular}{|c|c|c|c|c|c|c|c|}
\hline \multirow[t]{2}{*}{ Config. no } & \multicolumn{3}{|c|}{ Reference image } & \multicolumn{3}{|c|}{ Deformed image } & \multirow[t]{2}{*}{$\mathrm{C}$ (pixels } \\
\hline & Source & Component & Wavelength $(\mu \mathrm{m})$ & Source & Component & Wavelength $(\mu \mathrm{m})$ & \\
\hline 1 & White & Lens Filter & 1.31 & White & Lens Filter & 1.55 & 2.92 \\
\hline 2 & Laser & Lens Filter & 1.31 & Laser & Lens Filter & 1.55 & 2.96 \\
\hline 3 & Laser & Lens & 1.31 & Laser & Lens & 1.55 & 0.33 \\
\hline 4 & Laser & Lens & 1.31 & Laser & Lens Filter & 1.31 & 1.47 \\
\hline 5 & Laser & Lens & 1.55 & Laser & Lens Filter & 1.55 & 1.80 \\
\hline
\end{tabular}



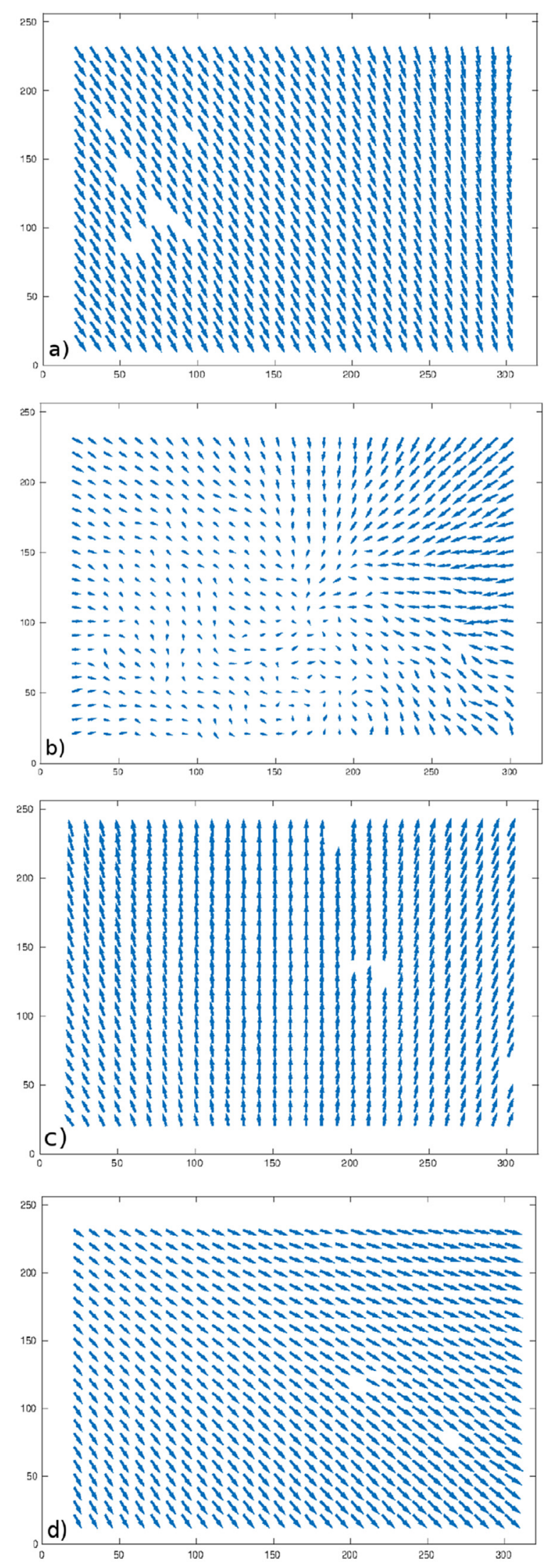

23

Fig. 4. Displacement maps for: a) configuration 1 ; b) configuration 3; c) configuration 4 ; d) configuration 5 . provided in this part for a camera-lens-filter system. At its maximum it reaches almost 3 pixels (configuration 2), and is mainly caused by the filters.

\subsubsection{Reproducibility of measurement}

A repetition test is performed to evaluate the precision of previous DIC measurements. As those displacements are supposed to be applied to infrared images in the thermography step, it is important to prove that there is no artifacts such as component unstability in the whole process. The optical component which could be affected by it are the filters. They are moved by the filterwheel, and this section studies the possible variation of the tilt between the lens and the filter.

To do so, a reference image at $1.31 \mu \mathrm{m}$ is acquired, the filter wheel goes to the $1.55 \mu \mathrm{m}$ filter location, another image is acquired and the displacement is calculated. Then the filterwheel is moved to another location, then replaced at $1.55 \mu \mathrm{m}$ location, another image is acquired and another displacement map is calculated. This test is repeated until ten displacement maps are obtained (or 19 rotations of the filter wheel). The idea is then to analyse the displacement maps variation over the ten tests. The displacements provided by VIC2D software are given along $\mathrm{u}$ and $\mathrm{v}$ direction (du and dv, see Eq. (3)), so it is possible to calculate a standard deviation map over the ten tests $\left(\sigma_{d u}\right.$ and $\left.\sigma_{d v}\right)$, and their mean values over the image $\left(\overline{\sigma_{d u}}\right.$ and $\left.\overline{\sigma_{d v}}\right)$. Criteria $\mathrm{C}$ is also calculated for each test and its standard deviation over the ten tests $\left(\sigma_{C}\right)$.

The results show a very small variation of the displacement maps, $\overline{\sigma_{d u}}$ is equal to 0.0037 pixels, $\overline{\sigma_{d v}}$ to 0.0055 pixels and $\sigma_{C}$ is around 0.0051 pixels. The system can thus be considered as temporarily stable, and the filters are positioned at an exact position after each rotation.

\section{Correction of thermal images}

\subsection{Protocol}

The correction protocol is divided into two steps. First, a chromatic aberration calibration is performed for the calculation of the reference displacement field between the bichromatic images. Next, the real-time correction is achieved by applying this reference displacement field to the thermal images that are used to compute the radiance temperature fields.

The chromatic aberration calibration resumes the experiment of the previous section according to configuration 1 . The reference calibration target is illuminated by the white source. The bichromatic images are the reference image acquired at the wavelength of $1.55 \mu \mathrm{m}$ and the distorted image acquired at the wavelength of $1.31 \mu \mathrm{m}$. As correlation is symmetrical, it has no influence on the distortion (except for the sign of the vectors). The displacement of the image at $1.31 \mu \mathrm{m}$ is then calculated referring to the one at $1.55 \mu \mathrm{m}$. Using DIC, the reference displacement field of the image at $1.31 \mu \mathrm{m}$ is then calculated referring to the one at $1.55 \mu \mathrm{m}$.

For the bichromatic measurement, the real-time correction consists in applying the image reference displacement field to the image acquired at $1.31 \mu \mathrm{m}$. The corrected image is the input for the calculation of the radiance temperature field $T_{R 1}(u, v)$. As the displacements are equal to a non-entire number of pixels, an interpolation step calculates new values of a pixel referring to its nearest four neighbours. After this interpolation step, the corrected image has the same number of pixels as the raw image. Residual blur will come from the interpolation step, which unavoidably induces an error of \pm 1 pixel. 


\subsection{Temperature error with chromatic aberration correction}

The colour temperature field is again calculated on the twoemissivity sample by a bichromatic thermography method including a chromatic aberration correction. Applying the previous protocol to the image at $1.31 \mu \mathrm{m}$, the corrected colour temperature is calculated. The colour temperature obtained for the parts outside the boundary are considered as the reference (true) temperature, i.e. $975 \mathrm{~K}$ for the $Y S Z: \mathrm{Er}^{3+}$ part, and $950 \mathrm{~K}$ for the $Y S Z: D y^{3+}$ part. A temperature error can then be calculated as the absolute difference between the colour temperature at a given pixel and its corresponding reference temperature. It is displayed in the semilogarithmic graph in Fig. 5, along the dashed line already presented, before and after correction.

This graph clearly shows the benefits of the chromatic aberration correction in terms of temperature error. Without correction, the error can be significant for the three pixels at the boundary of the two parts, as already shown in Fig. 2. With correction, the two boundaries are coincident and the calculation of colour temperature no longer shows any incoherent values, with the error staying within $10 \mathrm{~K}$. Thus, this correction has greatly improved the accuracy of the temperature measurement and shown that it can be a very useful tool for quantitative field temperature measurement.

\section{Conclusion}

This paper deals with the temperature error in polychromatic thermography measurement due to chromatic aberrations. The polychromatic thermography system studied was composed of a camera operating in the NIR spectral band, a lens and a set of filters. First, the temperature error was quantified on a specific sample composed of two emissivities. The suggested approach was based on a first calibration step, where the displacements of an image at one wavelength were calculated with reference to another wavelength. Then the displacement map was applied to the other spectral image. This correction greatly improved the accuracy of temperature readings.

The first section investigated the influence of chromatic aberration on the bichromatic temperature error. The chromatic aberration on the polychromatic thermography system leads to a pixel shift or displacement on radiance temperature measurements at different wavelengths. When these radiance temperatures are merged to establish a true temperature value, this spatial inconsistency leads to incorrect temperature values at the boundary of

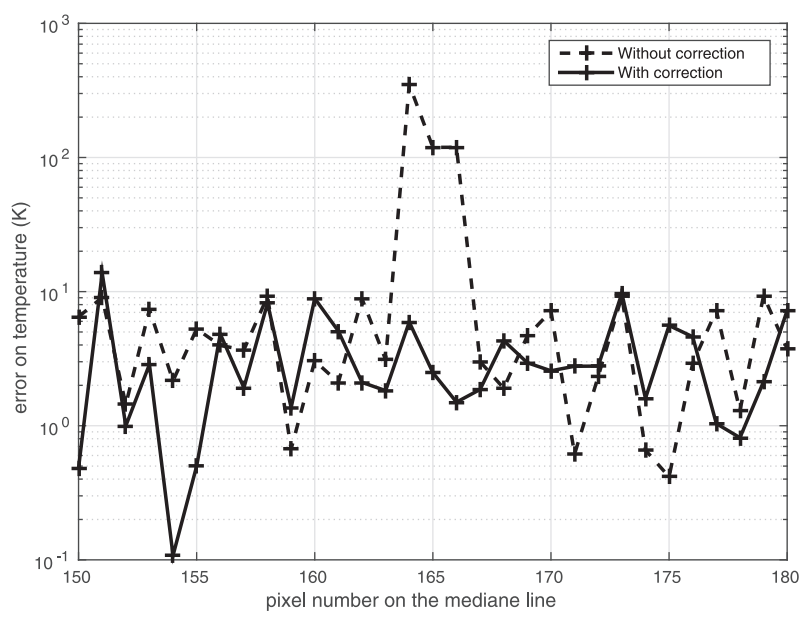

Fig. 5. Semi-log graph of the absolute temperature error before and after correction versus the pixel number. the two materials of the heterogeneous scene. Such errors can reach $40 \%$.

The next section presented the suggested approach for the software correction of chromatic aberration on temperature. The approach is based on the evaluation by DIC of two-dimensional displacement between the pixels for the different wavelengths. By tuning the experimental conditions (laser source, with or without filter), a dissociation of the effect of each optical element was deduced. The main source of distortion (almost 3 pixels) were the filters, probably due to the tilt between them and the lens.

The last section was devoted to the correction algorithm. The displacement was applied to the image at a first wavelength, taking the second one as a reference. As the displacement was equal to a non-entire number of pixels, a step of image interpolation was computed between the four neighbours. When this was applied to the temperature measurement application, the accuracy was greatly improved.

To conclude, for polychromatic thermography, the computation of true temperature field measurement needs to take into account the impact of chromatic aberration. The article suggests an approach based on Digital Image Correlation and a rigorous characterization. The main originality of this work is to prove that the correction leads to a significant improvement in the temperature measurement.

\section{References}

[1] Adomeit U, Ebert R. IR-dual-band-camera demonstrator: experimental assessment, practical applications. In: Proc. SPIE, 6737; 2007. 673703-67370311.

[2] Amiel S, Loarer T, Pocheau C, Roche H, Gauthier E, Aumeunier M-H, et al. 2D surface temperature measurement of plasma facing components with modulated active pyrometry. Rev Sci Instrum 2014;85(10).

[3] Bodnar J-L. Crack detection by stimulated infrared thermography. Eur Phys J Appl Phys March 2014;65:31001.

[4] Boult TE, Wolberg G. Correcting chromatic aberrations using image warping. In: Computer vision and pattern recognition, 1992. Proceedings CVPR'92., 1992 IEEE computer society conference on; Jun 1992. p. 684-7.

[5] Dusserre G, Nazaret F, Robert L, Cutard T. Applicability of image correlation techniques to characterise asymmetric refractory creep during bending tests. J Eur Ceram Soc 2013;33(2):221-31.

[6] Fu T, Liu J, Tang J, Duan M, Zhao H, Shi C. Temperature measurements of hightemperature semi-transparent infrared material using multi-wavelength pyrometry. Infrared Phys Technol 2014;66(0):49-55.

[7] Gilblas R, Sentenac T, Hernandez D, Le Maoult Y. Quantitative temperature field measurements on a non-gray multi-materials scene by thermoreflectometry. Infrared Phys Technol 2014;66(0):70-7.

[8] Giri LI, Tuli S, Sharma M, Bugnon P, Berger H, Magrez A. Thermal diffusivity measurements of templated nanocomposite using infrared thermography. Mater Lett 2014;115(0):106-8.

[9] Horn Berthold K, Schunck Brian G. Determining optical flow. In: Proc. SPIE, 0281; 1981. p. 319-31.

[10] Irie K, McKinnon AE, Unsworth K, Woodhead IM. A technique for evaluation of ccd video-camera noise. 2008.

[11] Kang SB. Automatic removal of chromatic aberration from a single image. In: Computer vision and pattern recognition, 2007. CVPR'07. IEEE conference on; June 2007. p. 1-8.

[12] Kaufmann V, Lasdstter R. Elimination of color fringes in digital photographs caused by lateral chromatic aberration. In: CIPA XX Intl. symposium; September 2005.

[13] Krapez JC. Radiative measurements of temperature. In: Thermal measurements and inverse techniques. Boca Raton, FL: CRC Press, Taylor And Francis; 2011. p. 185-230.

[14] Luhmann T, Hastedt H, Tecklenburg W. Modelling of chromatic aberration for high precision photogrammetry. In: IAPRS, vol. XXXVI; September 2006. p. 173-8. Part 5.

[15] Mallon J, Whelan PF. Calibration and removal of lateral chromatic aberration in images. Pattern Recognit Lett 2007;28(1):125-35.

[16] Orteu J-J. 3-d computer vision in experimental mechanics. Opt Lasers Eng 2009;47(3-4):282-91. Optical Measurements.

[17] Rehm R, Walther M, Fleiner J, Schmitz J, Ziegler J, Cabanski W, et al. Bispectral thermal imaging with quantum-well infrared photodetectors and InAs/GaSb type-II superlattices. In: Proc. SPIE, 6206; 2006. 62060Y-62060Y-11.

[18] Rodiet C, Rémy B, Degiovanni A, Demeurie F. Optimisation of wavelengths selection used for the multi-spectral temperature measurement by ordinary least squares method of surfaces exhibiting non-uniform emissivity. Quant 
InfraRed Thermogr J 2013;10(2):222-36.

[19] Siroux M, Kasem H, Thevenet J, Desmet B, Dufrenoy P. Local temperatures evaluation on the pindisc interface using infrared metrology. Int J Therm Sci 2011;50(4):486-92.

[20] Suhre DR, Denes LJ, Gupta N. Telecentric confocal optics for aberration correction of acousto-optic tunable filters. Appl Opt Feb 2004;43(6):1255-60.

[21] Sentenac T, Gilblas R, Hernandez D, Le Maoult Y. Bi-color near infrared thermoreflectometry: a method for true temperature field measurement. Rev thermoreflectometry: a method for true temperature
Sci Instrum Dec 2012;83(12). $124902-124902-14$.

[22] Sentenac T, Gilblas R. Noise effect on interpolation equation for near infrared thermography. Metrologia 50, Mar 2013:208.

[23] Strong J. Achromatic doublet lenses for infrared radiation. Appl Opt Jun 1971;10(6):1439-43.

[24] Sutton MA, Wolters WJ, Peters WH, Ranson WF, McNeill SR. Determination of displacements using an improved digital correlation method. Image Vis Comput 1983;1(3):133-9.

[25] Wang X. Intelligent multi-camera video surveillance: a review Pattern Recognit Lett 2013;34(1):3-19. Extracting Semantics from Multi-Spectrum Video. 